Acta Cryst. (2002). A58 (Supplement), C78

9,10-PHENANTHRENEQUINONE: NOT YOUR AVERAGE STRUCTURE

A.D. Rae A.C. Willis

Research School of Chemistry Australian National University CANBERRA ACT 0200 AUSTRALIA

9,10-phenanthrenequinone, $\mathrm{C}_{14} \mathrm{H}_{8} \mathrm{O}_{2}$, is both polymorphic and polytypic. The beta form allows different stackings of ordered layers perpendicular to $\mathrm{c}^{*}$. These are best understood as different occupancy wave modulations of a 1:1 disordered parent structure in space group $P 21 / c$ in which most atoms overlap. Ordered layers have $C-1$ symmetry in a cell with the a and $\mathrm{b}$ axes doubled compared to the parent structure. Each layer has two possible origins.

Different stacking sequences allow two orientations of a $\mathrm{Z}=8, C c$, structure and two orientations of a $\mathrm{Z}=16, F-1$ structure with the $\mathrm{c}$ axis now doubled. It was found that for some crystals it is necessary to model the reflection data assuming uncorrelated mosaic blocks of different space group symmetry as well as orientation. To allow for stacking faults a disordered component with the parent symmetry was included. Data selection allowed reliable geometry for both the $C c$ and $F-1$ components to be obtained using standard programs. The alpha form allows different stackings of ordered layers perpendicular to $\mathrm{a}^{*}$. These are best understood as different occupancy wave modulations of a 1:1 disordered parent structure in space group Pnmn in which most atoms overlap. Ordered layers have $P 2 / c$ symmetry and each layer has two possible origins.

Different stacking sequences allow two orientations of a $\mathrm{Z}=4, P 21 \mathrm{cn}$ structure and two orientations of a $\mathrm{Z}=8, B 2 / c$ structure with double the a axis (a non standard setting of $P 2 / c$ ).

Keywords: POLYTYPISM POLYMORPHISM REFINEMENT

Acta Cryst. (2002). A58 (Supplement), C78

\section{MULTIPLE ROTATION FUNCTION}

A. Urzhumtsev L. Urzhumtseva

${ }^{1}$ LCM3B, UMR 7036 CNRS, Universite H. Poincare, Nancy 1, Vandoevre-lesNancy, France

A simultaneous analysis of several rotation functions allows to identify the model orientation when a single rotation function fails to provide with the answer. These functions can be obtained by a usual modification of the model or by variation of the resolution at which the function is calculated. A specially suited case is a knowledge of several NMR models.

An algorithm has been developed in order to compare multiple rotation functions. All rotation peaks coming from several rotation functions are taken together and the orientations which are closer each to other than a chosen closeness limit are considered as 'to be the same within the given limit'. The size of all such groups (clusters) of similar orientations can be calculated and shown in a diagram; the cluster corresponding to the correct orientation is as a rule the mostly populated and therefore the analysis of the size of clusters allows to find the model orientation.

The algorithm has been realized in a corresponding user-friendly interactive computer program COMPANG. The same program allows to study the orientations related by a non-crystallographic symmetry. Inversely, the known non-crystallographic rotation axis can reinforce the signal in the multiple rotation function. When the orientation of the model becomes available, its position can be found much easier; low resolution data can essentially help in such a search. A number of difficult cases of the molecular replacement can be solved by new means discussed in the article; several such examples are discussed.

Keywords: ROTATION FUNCTION, MOLECULAR REPLACEMENT, CLUSTER ANALYSIS
Acta Cryst. (2002). A58 (Supplement), C78

\section{RECONSTRUCTION OF XRAY IMAGES FROM SPECKLE PATTERNS BY ITERATION}

J. Spence H. He M. Howells S. Marchesini U. Weierstall G. Hembree Lawrence Berkeley Laboratory 1 Cyclotron Rd Ms 7-222 BERKELEY CA 94720 USA

Soft X-ray transmission diffraction patterns have been obtained from micronsized objects at $550 \mathrm{eV}$ and $30 \mathrm{~nm}$ resolution. These speckle patterns are inverted to form real-space images, using the Fienup Hybrid Input-Output algorithm to solve the phase problem. The design of a holography chamber with zone-plate monochromator is described, and experimental conditions for lensless imaging are reviewed. Experimental results using laser light and coherent electrons are also demonstrated, together with simulations. Resolution limits and extensions to tomography are discussed.

References:

Weierstall et al Ultramic. 2002 in press, and Spence et al, Phil Trans. 2002. in press.

\section{Keywords: SOFT X-RAY IMAGING}

Acta Cryst. (2002). A58 (Supplement), C78

DIFFERENCE PATTERSON MAPS FOR IMPROVING MANY-BODY SEARCHES IN MOLECULAR REPLACEMENT

C. Alvarez-Rua J. Borge S. Garcia-Granda

Departamento de Quimica Fisica y Analitica. Facultad de Quimica. Universidad de Oviedo. C/ Julian Claveria, 8. 33006 Oviedo. SPAIN.

In spite of significant improvements of algorithms in the last few years, there are still numerous cases that cause the molecular replacement method to fail. One of these is the presence of various molecules in the asymmetric unit of the crystal cell. In this context, the incorporation of known structural information has proved to enhance the discrimination of a rotation function evaluated in Patterson space when the orientation of many independent molecules has to be determined. The rotation procedure is based in the application of a vector search algorithm specially designed for macromolecular crystallography. The basis of this procedure is the comparison between an observed Patterson map from the target structure, which can be considered as a representation of all the interatomic vectors in the unit cell translated to the origin and a vector set from the search model. The degree of fit between them is obtained through the evaluation of an Image Seeking Function, that attains a maximum at the correct orientation of the search model. When many independent molecules are present in the asymmetric unit of the crystal cell, the performance of this rotation function can be improved by subtraction of the contributions of already positioned molecules from the observed Patterson map. A new computer program, OVIDIF (1), has been designed to compute the difference Patterson maps which are used as input data by the rotation function program OVIONE (2).

References

(1) Alvarez-Rua, C., Borge, J. \& Garcia-Granda, S. (2002). Acta Cryst. D58, 215-224. (2) Borge, J., Alvarez-Rua, C. \& Garcia-Granda, S. (2000). Acta Cryst. D56, 735-746.

Keywords: MOLECULAR REPLACEMENT, ROTATION FUNCTION, MANY-BODY SEARCHES 